\title{
Study of Ring-to-chain Structural Change of Evaporated Amorphous Selenium by Reverse Monte Carlo Simulation
}

\author{
${ }^{*}$ Hitoshi SAITO, Kazuhisa TAKETOSHI and Kaoru KOZU \\ *Department of Electrical Engineering, Faculty of Engineering, Kokushikan University, \\ 4-28-1 Setagaya, Setagaya-ku, Tokyo, Japan
}

(Received October 10, 2000, Accepted April 7, 2001)

\begin{abstract}
We studied the photoinduced ring-to-chain structural change of amorphous selenium (a-Se) evaporated at room temperature with in situ observation by highly sensitive electron diffraction and Reverse Monte Carlo (RMC) simulation. Although the structure before irradiation (SBI) is a random chain structure containing random rings (RCSR) ( $30 \%$ atoms in relevant number of 1013 network atoms), the structure after irradiation (SAI) is a random chain structure. These are obtained with the RMC simulation of the difference in structure factors from RCSR. The average bond angles for SBI and SAI are $105.1^{\circ}$ and $104.3^{\circ}$, respectively. The average dihedral bond angles for SBI and SAI are $101.7^{\circ}$ and $100.7^{\circ}$, respectively. Rings that form the initially closed loops in SBI are broken by photoirradiation. Bonds of random rings in SAI are not closed, and change to the random chain structure. These results are similar to those of Roy et al. (Phil. Mag. B, 78, 1, 1998, 87-94.) obtained by extended X-ray absorption fine structure spectroscopy (EXAFS).
\end{abstract}

\section{Introduction}

Recently, Roy et al. reported the photoinduced ring-to-chain structural change of an amorphous selenium (a-Se) film evaporated at substrate room temperature with extended X-ray absorption fine structure spectroscopy (EXAFS) ${ }^{1)}$. They reported that a-Se before irradiation has closed rings that change to successive chains after irradiation. The present authors also observed the same phenomenon by means of in situ observation using a highly sensitive improved electron diffractometer, and obtained structural images using the Reverse Monte Carlo (RMC) simulation of the difference in structure factors, which was reported by McGreevy and Howe ${ }^{2)}$. These findings have not yet been reported. Here, we report the experimental results and the obtained structure.

\section{RMC method}

The specimens were prepared by thermal evaporation of bulk Se powder of $99.9999 \%$ purity in a vacuum of $2 \times 10^{-4} \mathrm{~Pa}$ on calcium fluoride $\left(\mathrm{CaF}_{2}\right)$ maintained at room temperature. The diffraction pattern was taken in real time every $1 / 5 \mathrm{sec}$ throughout the evaporation process. The fluorescent screen was composed of transparent evaporated $\mathrm{ZnS}: \mathrm{Ag}$, which had no granularity. The detector is a charge coupled device (CCD) of 480000 pixels.
Shot noise of the diffraction pattern could be reduced by averaging them along a circular locus of equal radius. After the a-Se film used for the observation of the first diffraction pattern was irradiated by a $500 \mathrm{~W}$ xenon lamp, the second diffraction patterns were taken immediately afterward in the same vacuum.

The main points of RMC simulation are outlined as follows ${ }^{2}$. If $Q=4 \pi \sin (\theta) / \lambda$ and $\rho$ is the density, the calculated atomic number per unit volume $g_{c}$ is described by

$$
g_{\mathrm{c}}(r)=n(r) /\left(4 \pi r^{2} \mathrm{dr} \rho\right),
$$

where $\theta, \lambda, n(r), r$ and dr are the Bragg angle, the wavelength, the number of atoms per dr, the spherical radius from the center and the width of the sphere, respectively. From eq. (1), the radial distribution function $(R D F)$ is described by

$$
R D F=4 \pi r^{2} g_{\mathrm{c}}(r) \text {. }
$$

The structure factor $A_{c}(Q)$ is described by

$$
A_{c}(Q)-1=\rho \int 4 \pi r^{2}\left(g_{\mathrm{c}}(r)-1\right) \sin (Q r) /(Q r) \mathrm{dr} .
$$

The difference $D i$ for observed and calculated structure factors is described by

$$
D i=\sum\left(A_{o}\left(Q_{i}\right)-A_{c}\left(Q_{i}\right)\right)^{2} / \sum A_{o}\left(Q_{i}\right)^{2},
$$

where $D i$ is a reliability factor, $A o$ is the observed structure factor and the summation is taken over Qi. 
Hereafter, a correction is added to the coordinate of the i-th atom $\left(x_{i}, y_{i}, z_{i}\right)$ if $D i$ after the calculation is less than that before the calculation. These techniques are the same as those of the conventional least squares method.

\section{Difference in structure factors}

If $g_{c}(r)$ in eq. 1 is separated into two parts as $(1-p) g 1_{c}(r)$ and $p g 2_{c}(r)$ ( $p$ : atomic composition ratio), the structures of which are independent without correlation in the coherent diffraction, then the following equations stand:

$$
\begin{aligned}
& g_{c}(r)=(1-p) g 1_{c}(r)+p g 2_{c}(r), \\
& g 1_{c}(r)=n 1(r) /\left(4 \pi r^{2} \operatorname{dr} \rho\right), \\
& g 2_{c}(r)=n 2(r) /\left(4 \pi r^{2} \operatorname{dr} \rho\right),
\end{aligned}
$$

where $n 1(r)$ and $n 2(r)$ are atomic numbers in the two parts, respectively.

From eq. 3 the following equations stand: $A 2_{c}(Q)-1=\rho \int 4 \pi r^{2}\left(g 2_{c}(r)-1\right) \sin (Q r) /(Q r) \mathrm{dr}$,

$$
p A 2_{\mathrm{c}}(Q)-p=\rho \int_{\times \sin (Q r) /(Q r) \mathrm{dr} .} 4 \pi r^{2}\left(p g 2_{c}(r)-p\right)
$$

In the same reason as eq. 9 ,

$$
\begin{aligned}
& (1-p) A 1_{\mathrm{c}}(Q)-(1-p) \\
& \quad=\rho \int_{\times \sin } 4 \pi r^{2}\left((1-p) g 1_{\mathrm{c}}(r)-(1-p)\right) \\
& \quad(Q r) \mathrm{dr},
\end{aligned}
$$

stand.

By the summation of eq. 9 and 10 ,

$$
\begin{aligned}
& p A 2_{c}(Q)+(1-p) A 1_{c}(Q)-1 \\
& \quad=\rho \int 4 \pi r^{2}(X) \sin (Q r) /(Q r) \mathrm{dr}, \\
& X=(1-p) g 1_{c}(r)+p g 2_{c}(r)-1,
\end{aligned}
$$

are obtained.

From eq. 5 and 3,

$$
\begin{aligned}
& X=g_{c}(r)-1, \\
& A c(Q)=p A 2_{c}(Q)+(1-p) A 1_{c}(Q), \\
& A_{c}(Q)-(1-p) A 1_{c}(Q)=p A 2_{c}(Q),
\end{aligned}
$$

are obtained, where $A 1_{c}(Q)$ and $A 2_{c}(Q)$ are structure factors of $g 1_{c}(r)$ and $g 1_{c}(r)$, respectively.

Figure 1 shows the a-Se structure factors before and after irradiation. We discovered unexpressed features related to the difference between the two structure factors before and after irradiation.

Figure 2 shows the relationship between $Q$ and the structure factor difference for $p$ ranging from $0.0 \sim 1.0$. The letter $p$ in Fig. 2 shows $(A c-(1-p)$ $A c 1)+n$, where $n, A c$ and $A c 1$ are the integer number for level shift, those of SBI and SAI, respectively. Figure 3 shows the difference in structure factors before and after irradiation and the structure factors of random rings and chains. The definition

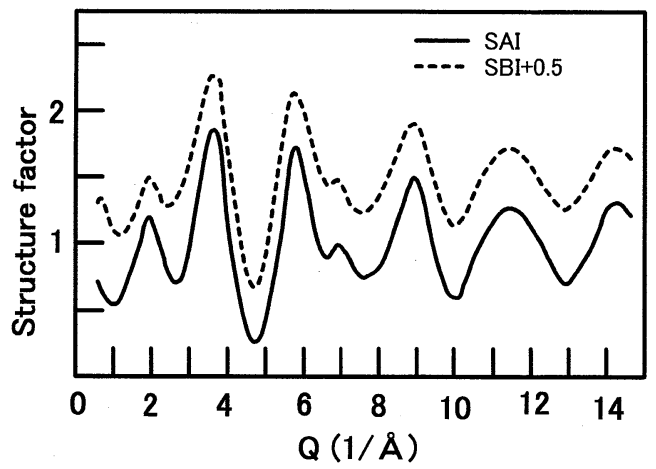

Fig. 1 a-Se structure factors before and after irradiation.

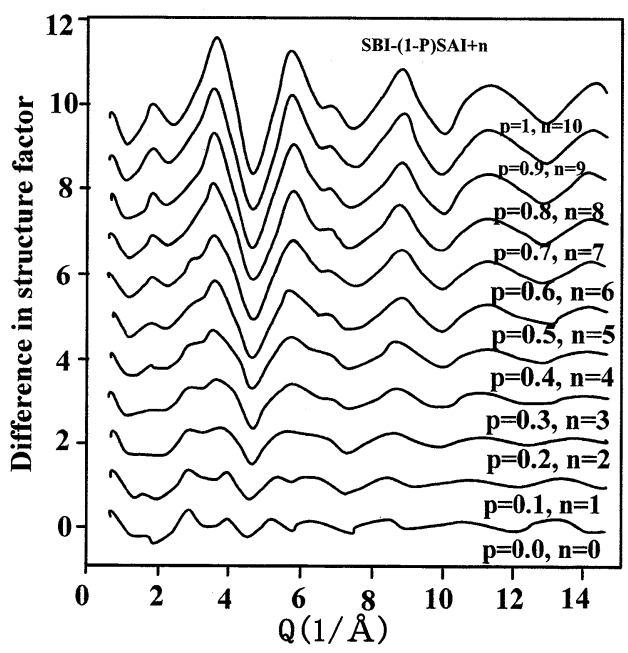

Fig. 2 Relationships between $Q$ and the structure factor before and after irradiation for $p$ ranging from $0.0 \sim 1.0$.

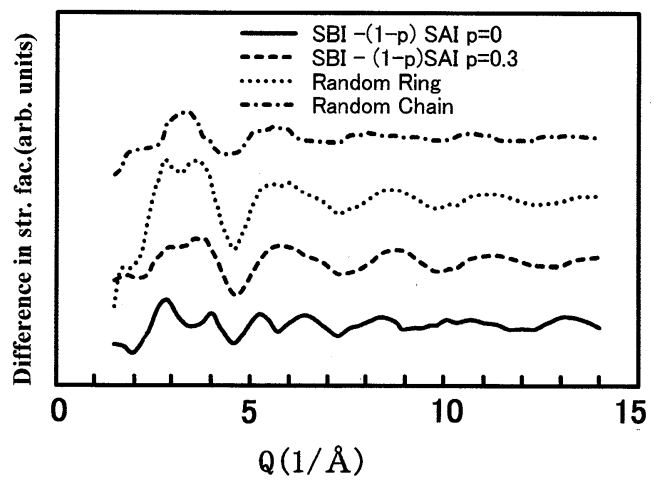

Fig. 3 Difference in structure factors before and after irradiation and the structure factors of random rings and chains. 


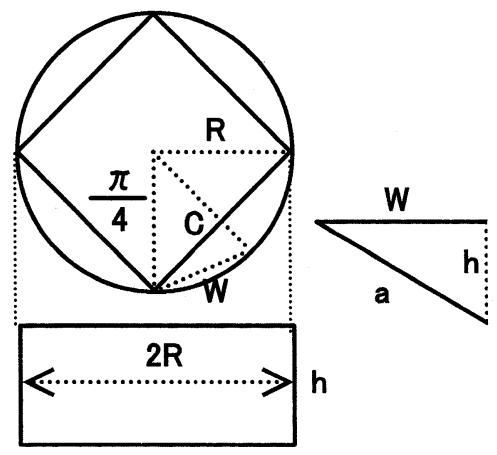

Fig. 4 Definition of a random ring structure.

of random rings and chains is given below. The difference of $p=0$ initially appears meaningless. However, the difference of $p=0.3$ is similar to that of the random ring structure, and not the random chain structure shown in Fig. 3. We consider these findings to be not an accidental coincidence but a logical consequence. In other words, the a-Se structure before irradiation contains partially random rings $(30 \%$ atoms in a relevant number of network atoms of 1013) and the remaining structure is almost the same after irradiation, although a-Se is not thought to contain rings.

\section{Random ring structures}

A random ring structure is illustrated in Fig. 4. Eight coordinates $\left(R_{\mathrm{i}}\right)$ are written as

$$
\begin{aligned}
& R 1=(0, R, h), \\
& R 2=(X, Y, 0), \\
& R 3=(R, 0, h), \\
& R 4=(X,-Y, 0), \\
& R 5=(0,-R, h), \\
& R 6=(-X,-Y, 0), \\
& R 7=(-R, 0, h), \\
& R 8=(-X, Y, 0), \\
& h=a \cos \left(\theta / 2^{\circ}\right),
\end{aligned}
$$

where $R$ is $C / \operatorname{sqr}(2), X$ is $R \cos (\pi / 4)$ and $Y$ is $R$ $\sin (\pi / 4)$.

A random chain structure is illustrated in Fig. 5, which is the same as the disordered chains of Misawa and Suzuki ${ }^{4)}$. The length of the secondary adjacent atom in the random rings and chains is $3.47 \AA$, similar to that in the crystalline structure. In the generation of actual random rings, the mean and the standard deviation of $a$, of which distribution is Gaussian, are $2.33 \AA$ and $0.17 \AA$, respectively, and the mean and the standard deviation of $\theta$, of which distribution is Gaussian, are $106.3^{\circ}$ and $5.0^{\circ}$, respectively. The starting model of 1013 atoms is made as follows. First the $30 \%$ random rings, the

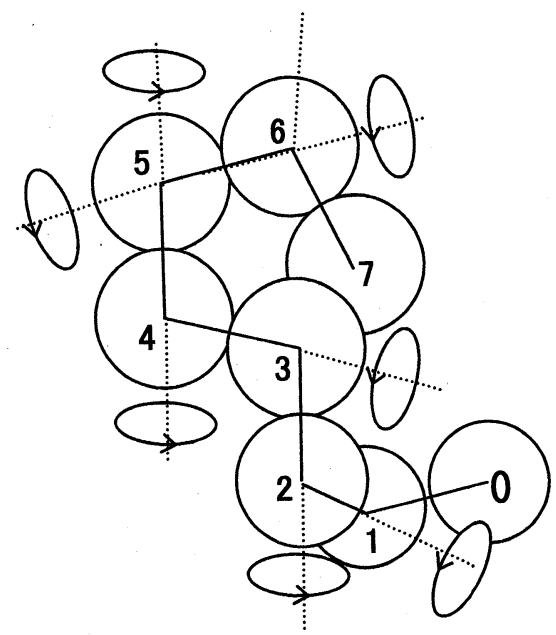

Fig. 5 Definition of a random chain structure.

atomic number of which is $304(8 \times 38)$, are generated in a sphere of radius $19.42 \AA$. In the generation, the fundamental random ring explained from eq. 16 to eq. 24 is spun by certain degrees within $\pm 180^{\circ}$ along the $x$-axis, $y$-axis and $z$-axis, and scattered so that the inter-ring distance is more than 3.47 A. Second, the remaining space of the sphere is filled with the random chains so that the interring and inter-chain distances is greater than 3.47 $\AA$. The atomic density is 0.033 (atoms/unit volume), which is the same as that of crystalline selenium.

\section{Details of RMC calculation}

We studied the amorphous structure by RMC simulation $^{2}$. The convergent algorithm in our RMC simulation is slightly different from the orthodox one ${ }^{3)}$. It resembles a diagonal matrix approximation of the least squares in the early stage of crystalline structural analysis. The total atomic number is chosen to be 1013 in order to decrease the statistical error of a small sample. In the $j$-th run, assume that the $x, y$ and $z$ components of the atomic coordinate of the $i$-th atom $\left(r_{i j}\right)$ are given by $\left(x_{i j}, y_{i j}, z_{i j}\right)$, and that the following displacement is done reciprocally:

$$
\begin{aligned}
& x_{i j}=x_{i j-1}+r a^{*} r_{\max }, \\
& y_{i j}=y_{i j-1}+r a^{*} r_{\max }, \\
& z_{i j}=z_{i j-1}+r a^{*} r_{\max },
\end{aligned}
$$

where $r a$ is the appropriate initial value $(0.25 \AA)$, and $r_{\max }$ is the decreasing weight, the initial value of which is unity. In the above equation, if the displacement of eq. 25 is generated, all components except for $x_{i j}$ are left to be the original ones. If $D_{i j}<$ 


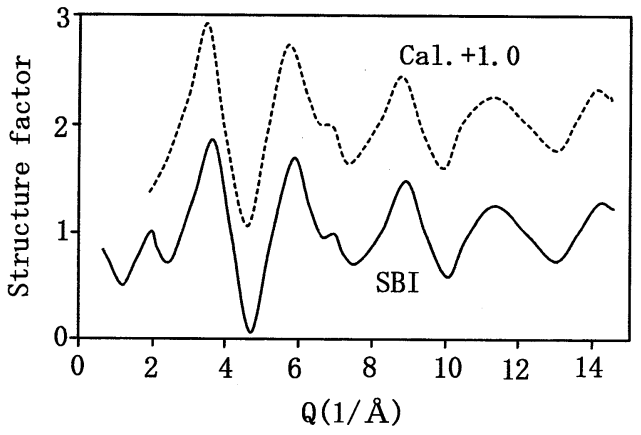

Fig. 6 Structure factor in SBI for the calculated and observed values.

$D_{i j-1}$ in this operation, then $x_{i j}$ is memorized for coordinate correction. If $D_{i j}>D_{i j-1}$ in this operation, then $x_{i j}, \mathrm{y}_{\mathrm{ij}}$ and $z_{i j}$ are memorized for coordinate correction as follows:

$$
\begin{aligned}
& x_{i j}=x_{i j-1}-r a^{*} r_{\max }, \\
& y_{i j}=y_{i j-1}-r a^{*} r_{\max }, \\
& z_{i j}=z_{i j-1}-r a^{*} r_{\max } .
\end{aligned}
$$

Moreover, at the end of these operations, the actual reliability factor $(D a j)$ is searched for all memorized corrected values of $r_{i j}(1 \leqq i \leqq 1013)$ in the $j$-th run. If $D a j<D a j_{-1}$, then the original $r_{i j}$ values are replaced with the memorized $r_{i j}$, and $r_{\max }$ is replaced with $0.8^{*} r_{\text {max }}$. If $D a j>D a j_{-1}$, then, the original $r_{i j}$ values are maintained, and $r_{\max }$ is replaced with $0.8^{*} r_{\max }$. The repetition number of $j$ is 30 runs. In the above repetition, the decreasing weight maintained a constant ratio. If the decreasing weight was a constant difference value (constant step) of $\left(r_{\max }-0.1\right)>0$, the same convergent values of $r_{i j}$ as those in the constant ratio were obtained. In addition to these convergent conditions, if the decreasing weight was a random value of $r_{\max , j-1}$, which was replaced with $r_{\max , j}\left(r_{\max , j-1}>\right.$ $\left.r_{\max , j}\right)$, the same convergent values of $r_{i j}$ were obtained. The calculated data in the following section were obtained by the constant weight ratio of the minimum reliability factor. The workstation used was that of DEC alpha of $600 \mathrm{MHz}$ and 64 bit. The computation language used was digital visual Fortran.

\section{Structural configuration}

We studied the structural differences before and after irradiation by RMC simulation. ${ }^{2)}$ We used the starting model before irradiation that contained $30 \%$ random rings and $70.0 \%$ random chains, namely, we obtained the structure before irradiation (SBI) from the random chain structure containing $30 \%$ random rings (RCSR). Second, we ob-

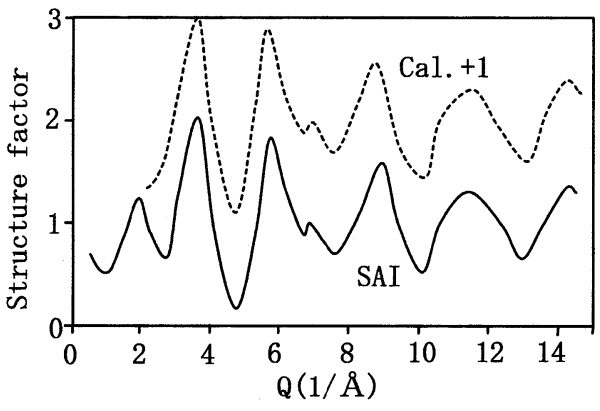

Fig. 7 Structure factor in SAI for the calculated and observed values.

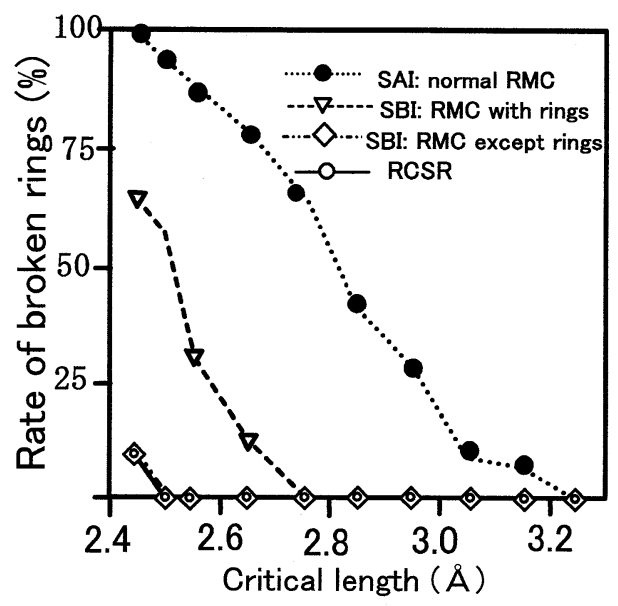

Fig. 8 Critical length and the rate of broken rings.

tained the structure after irradiation (SBI) from the RCSR and the structure after irradiation (SAI) from SBI. Figure 6 shows the relationship between the calculated and observed values of the structure factors in SBI. Figure 7 shows the calculated and observed values of the structure factors in SAI. The coincidences between the calculated and observed structure factors in SBI and SAI are very good. The reliability factors in SBI and SBI are $4.5 \%$ and $5.3 \%(2.0<Q<14.6)$, respectively.

RMC simulation of SBI is applied to the atoms except random rings. Figure 8 shows the relationship between the critical length and the rate of broken rings. White circles show the results for RCSR, diamonds show those for SBI, triangles show those for SBI which is applied to all the atoms of RCSR, and black circles show those for SAI. The rate of broken rings of SBI is the same as that of RCSR. The reliability factor is $4.5 \%$. These results support the existence of random rings. 


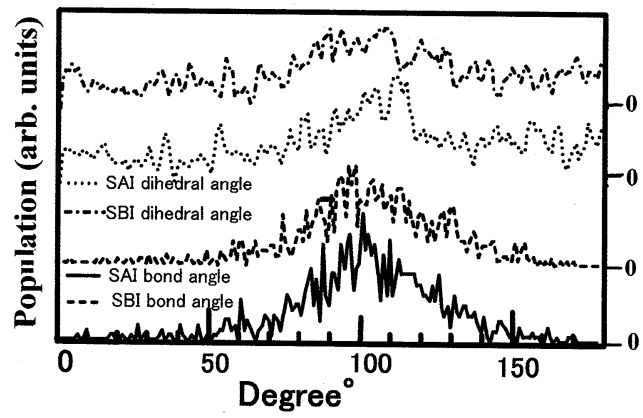

Fig. 9 Population distributions of bond and dihedral angles for SBI and SAI.

Moreover, if the RMC simulation of SBI is applied to all the atoms of RCSR, then the rate is zero above $2.75 \AA$ and the rate of broken rings is lower than that of SAI. This indicates that the structure factor of SBI itself contains random rings and differs from that of SAI, although they seems to resemble each other at a glance.

The population distributions of bond and dihedral angles for SBI and SAI are shown in Fig. 9. The average bond angles of SBI and SAI are $105.1^{\circ}$ and $104.3^{\circ}$, respectively, and are almost the same as those for trigonal $\mathrm{Se}\left(103.1^{\circ}\right)$ and alphamonoclinic $\mathrm{Se}\left(105.3^{\circ}\right)$. The results are similar to those reported by Shimojo et al.5) and Bichara and Pellegatti. ${ }^{6}$ ) The average dihedral bond angles of SBI and SAI are $101.7^{\circ}$ and $100.7^{\circ}$, respectively, and are almost the same as those for trigonal $\mathrm{Se}$ $\left(101.0^{\circ}\right)$, and alpha-monoclinic Se $\left(101.8^{\circ}\right)$.

Figure 10 shows the ring-to-chain change of the obtained structure for SBI and SAI.

Rings forming initially closed loops in SBI are broken by photoirradiation, and finally change to the chain structure in SAI. These results are similar to those of Roy et al. obtained by EXAFS and Raman scattering ${ }^{11}$.

\section{Conclusions}

We studied the photoinduced structural change of a-Se evaporated at room temperature with in situ observation by highly sensitive electron diffraction and RMC simulation of the difference in structure factors.

a) SBI is an RCSR, while SAI is a random chain structure.

b) The mean bond angles of SBI and SAI are $105.1^{\circ}$ and $104.3^{\circ}$, respectively, and are almost the

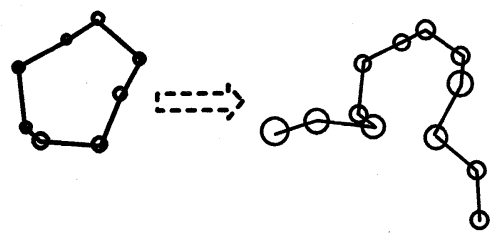

Ring in SBI Chain in SAI

Fig. 10 Ring-to-chain change of the obtained structure for SBI and SAI.

same as those of trigonal Se $\left(103.1^{\circ}\right)$ and alphamonoclinic $\mathrm{Se}\left(105.3^{\circ}\right)$.

c) The average dihedral bond angles of SBI and SAI are $101.7^{\circ}$ and $100.7^{\circ}$, respectively, and are almost the same values of trigonal $\mathrm{Se}\left(101.0^{\circ}\right)$, and alpha-monoclinic $\mathrm{Se}\left(101.8^{\circ}\right)$.

d) SAI does not contain random rings. Rings forming initially closed loops in SBI are broken by photoirradiation. All bonds of random rings in SAI are not closed, and change to the random chain structure. These results agree with those obtained by EXAFS and Raman scattering.

The origin of random rings in the a-Se evaporated sample is attributable to the structure of incident a-Se vapor in the vacuum. Hereafter we plan to study incident selenium vapor, and to report our results in the future.

\section{Acknowledgements}

We would like to extend our sincere thanks to Dr. F. Andoh of the NHK Science and Technical Research Laboratory and Dr. Y. Shimomoto of Hamamatsu Photonics K.K. This work was partially supported by a Grant-in-Aid for Scientific Research from the Ministry of Education, Science, Sports and Culture of Japan.

\section{References}

1) A. Roy, A. Kolobov, H. Oyanagi and K. Tanaka, Phil. Mag. B, 78, 1, 1998, 87-94.

2) R. McGreevy and M. Howe, Phys. Chemi, 24, 1991, $1-12$.

3) S. Kugler, L. Putztai, L. Rosta, P. Chieux and R. Bellissent, Phy. Rev. B. 48, 10, 1993, 7685-7688.

4) M. Misawa and K. Suzuki, J. Phys. Soc. Jpn. 44, $1978,1612$.

5) F. Shimojo, K. Hoshino, M. Watabe and Y. Zempo, J. Phys: Cond. Mat. 10, 1998, 1199-1210.

6) C. Bichara and A. Pellegatti, Phy. Rev. 49, 10, March 1994, 6581-6586. 